\title{
REDEFINITION OF THE ANOMALY OF THE NITRATE-PHOSPHATE RATIO
}

\author{
By L. H. N. Cooper, D.Sc., F.I.C.
}

Assistant Chemist at the Plymouth Laboratory

The paper on the ratio of nitrogen to phosphorus in the sea (Cooper, I937) was written before it was realized that in phosphate determinations, using two $\mathrm{ml}$. of molybdate reagent per $100 \mathrm{ml}$. of water, the magnitude of the salt error correction factor is changed by the use of a spectral filter. Due to the resulting uncertainty it was felt better to use uncorrected figures for phosphate content rather than to risk a correction which might later prove to be wrong. We now know (Cooper, I938) that the factor, $1 \cdot 35$, can be applied to all Plymouth data given in the I937 paper and presumably to the results of the Dana and Discovery $I I$ there cited. The ideal ratio of $20: \mathrm{I}$, for nitrate-N/ phosphate-P expressed as milligram-atoms, therefore becomes $20: \mathrm{I} \cdot 35=\mathrm{I} 4 \cdot 8$, or, say, I5: I. Most of Redfield's figures are probably amenable to the same correction. The revised nitrate- $\mathrm{N} /$ phosphate-P ratio agrees even better with the mean ratio of the thirteen sets of nitrogen and phosphorus analyses of plankton taken off Plymouth, viz. I6.3.

I suggest therefore that the "anomaly of the nitrate-phosphate ratio" be redefined as the amount by which the nitrate-phosphate ratio differs from fifteen, each salt being expressed in milligram-atoms and phosphate being corrected for salt error by the appropriate factor (see Cooper, I938).

\section{REFERENCES}

COOPER, L. H. N., 1937. On the ratio of nitrogen to phosphorus in the sea. Fourn. Mar. Biol. Assoc., Vol. xxiI, pp. I77-82.

- 1938. Salt error in determinations of phosphate in sea water. Fourn. Mar. Biol. Assoc., Vol. xxiII, pp. I7I-8. 